\title{
Quantifying the Efficiency of Förster-Assisted Optical Gain in Semiconducting Polymer Blends by Excitation Wavelength Selective Amplified Spontaneous Emission
}

\author{
Longfei Wu, Feng Luo, Larry Lüer, Beatriz Romero, Jose Manuel Otón, Qi Zhang, \\ Ruidong Xia, Juan Cabanillas-Gonzalez
}

ABSTRACT: We study the correlation between Förster resonance energy transfer (FRET) and optical gain properties in conjugated polymer blends based on regioregular poly(3-hexylthiophene) (P3HT) and poly(9,9-dioctylfluorene-alt-benzothiadiazole) (F8BT). First, FRET dynamics are investigated with femtosecond transient absorption spectroscopy observing a sub-picosecond energy transfer from F8BT to P3HT (550 fs) at medium doping levels ( $40 \%$ wt $\mathrm{P} 3 \mathrm{HT}$ in $\mathrm{F} 8 \mathrm{BT}$ ). Amplified spontaneous emission (ASE) is then characterized in blends upon exciting predominantly the host and guest polymers, respectively. The corresponding density of absorbed photons at threshold conditions is compared upon host or guest photoexcitation as a method to quantitatively determine the FRET-assisted ASE efficiencies. We observe a reduction in ASE efficiency upon host photoexcitation of $20 \%$, in the best case, respect to guest photoexcitation. Our results confirm that even in strongly coupled host:guest mixtures delayed exciton population by energy transfer is subtle to losses ascribed to exciton-exciton annihilation.

KEYWORDS: amplified spontaneous emission; conjugated polymer blends; laser spectroscopy; energy transfer; amorphous materials
INTRODUCTION Semiconducting polymer mixtures enable to combine notable charge transport and emission properties characteristic of pristine conjugated polymers. ${ }^{1,2}$ In particular, conjugated polymer blends where host and guest polymers are coupled by Förster resonance energy transfer (FRET) offer potential advantages for lighting and optical gain applications. ${ }^{3,4}$ In these blends, photoexcitation placed on the host polymer with wider optical gap is transferred to a lower optical band gap guest-conjugated strand. Being a long-range interaction, FRET enables to tune spectrally the emission away from the host absorption at very low guest contents, thus reducing self-absorption effects and consequently enhancing amplified spontaneous emission (ASE) and lasing performance. ${ }^{5}$ In addition, FRET has also been used to achieve colour tunability by changing the type or the concentration of the guest species. Gupta et al. ${ }^{4,6}$ blended several red-emitting PPVs into green-emitting PPV derivatives to obtain very low ASE thresholds and remarkably reduced optical losses. Camposeo et al. ${ }^{7}$ reported on low-loss and highly polarized waveguides fabricated from greenemitting and blue-emitting fluorene-based copolymers.

In host:guest polymer-based optical amplifiers, FRET-assisted optical gain must overcome intrinsic exciton relaxation pathways in the host polymer. Owing to the large fluence necessary to induce ASE in optically pumped lasers and optical amplifiers, exciton-exciton annihilation is perhaps the main loss mechanism, having a severe effect on the population of excited states. $^{8,9}$ Its outcome depends strongly on film molecular packing which is in turn, controlled by polymer chemical structure and morphology. ${ }^{10,11}$ The enhancement of interchromophore distance in the solid state may contribute 
to reduce exciton-exciton annihilation, leading to an overall improvement in the optical gain properties of polymer optical amplifiers. ${ }^{12,13}$

In this work, we quantitatively determine the contribution from FRET to ASE as well as the magnitude of competing exciton losses in F8BT:P3HT polymer blends. Efficient red emission and optical gain from P3HT in this blend has been previously demonstrated. ${ }^{14,15}$ Pump-probe spectroscopy reveals a host:guest FRET taking place in timescales down to 550 fs. ASE losses competing with FRET are quantitatively assessed with excitation wavelength-dependent ASE measurements.

\section{EXPERIMENTAL}

Blend films were prepared upon mixing toluene solutions of $20 \mathrm{mg} \mathrm{mL}^{-1}$ P3HT (BASF Sepiolid P200, >98\% regioregularity, $M_{\mathrm{n}} \sim 10 \mathrm{~kg} \mathrm{~mol}^{-1}$ ) with F8BT (American Dye Source, $M_{\mathrm{n}}$ $\sim 33 \mathrm{~kg} \mathrm{~mol}^{-1}, M_{\mathrm{w}} \sim 217 \mathrm{~kg} \mathrm{~mol}^{-1}, \mathrm{PD}=6.6$ determined with size exclusion chromatography in $1,3,4-\mathrm{TCB}$ at $150{ }^{\circ} \mathrm{C}$; calibrated with PS standards) in different weight ratios (180 wt \% P3HT) and subsequent spin-cast of blend solutions at $2000 \mathrm{rpm}$ on pre-cleaned quartz substrates. Absorption and photoluminescence (PL) measurements were performed on a UV-vis Cary spectrophotometer and a Fluoromax 4 fluorimeter (Jobin-Yvon), respectively. The pump-probe setup consisted of a femtosecond Clark MXR regenerative amplifier as primary source delivering $120 \mathrm{fs}$ pulses at $775 \mathrm{~nm}$ and $1 \mathrm{kHz}$ repetition rate. The primary beam was split into pump and probe beams. The pump beam was doubled in frequency (387 nm), sent onto a computer controlled delay line, and subsequently focused onto the sample $(0.1 \mathrm{~m})$ $\mathrm{cm}^{-2}$ ). The probe beam was in turn focused on a sapphire plate to generate a supercontinuum and overlap with the pump spot on the sample. A prism spectrometer (Ingenieurbüro Stresing, Berlin, Germany) equipped with a double superposed Si - InGaAs array detector for VIS and NIR operating at single shot was employed to monitor the transmitted light through the sample as well as a reference probe pulse. Both channels allowed for acquisition of pump-induced absorption changes on the sample. Measurements were carried out in vacuum to prevent sample photo-degradation. For ASE measurements, the photoexcitation was provided by a Q-switched [Nd3+:YAG] (neodymium-doped yttrium aluminum garnet) laser which pumped an optical parametric oscillator, delivering pulses of $12 \mathrm{~ns}$ at $10 \mathrm{~Hz}$ repetition rate. The selected pump wavelengths were 450 and $530 \mathrm{~nm}$, concomitant with maximum absorption of host and guest, respectively. The pulse energy incident on the sample was adjusted by the insertion of calibrated neutral density filters into the beam path. An adjustable slit and a cylindrical lens were used to shape the laser beam into a narrow $400 \mu \mathrm{m} \times$ $4 \mathrm{~mm}$ stripe on the sample. At sufficient excitation intensities, the spontaneously emitted photons were amplified via stimulated emission. The output signal was collected and sent in free-space configuration to a SP-2558 Princeton Instruments (Acton Research) spectrometer equipped with a liquid $\mathrm{N}_{2}$ cooled deep-depleted CCD. ASE thresholds were
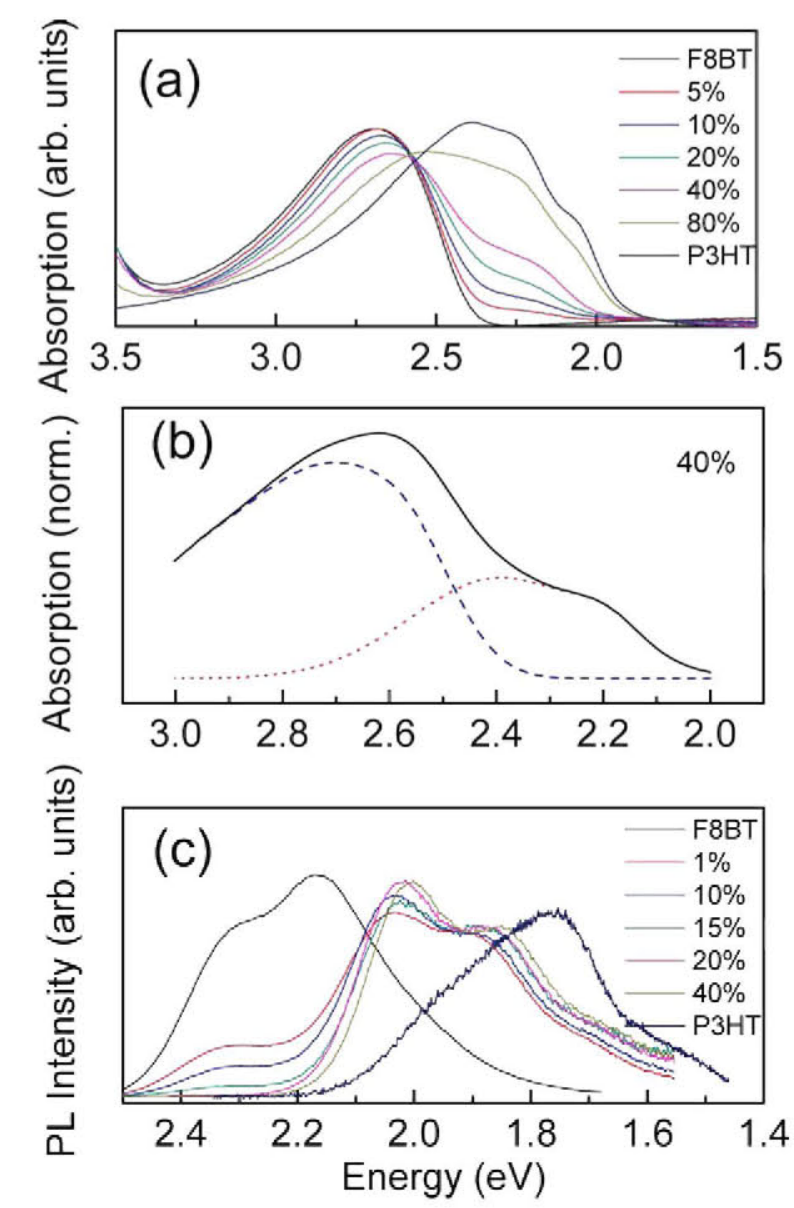

FIGURE 1 (a) Absorption of blends with 0, 5, 10, 20, 40, 80, and $100 \%$ weight fraction of $\mathrm{P} 3 \mathrm{HT}$. (b) Spectral decomposition of the absorption spectrum of a $40 \%$ blend into two Gaussian peaks corresponding to the F8BT (dashed) and F8BT:P3HT complex (dotted) contributions. (c) $\mathrm{PL}$ data are shown for blends containing $1,10,15,20$, and $40 \mathrm{wt} \% \mathrm{P} 3 \mathrm{HT}$ as well as for pristine F8BT and P3HT. Excitation wavelength was $450 \mathrm{~nm}$ (2.8 eV) for all samples except for pristine P3HT (530 nm, 2.3 $\mathrm{eV}$ ). [Color figure can be viewed in the online issue, which is available at wileyonlinelibrary.com.]

obtained as the incident pulse fluence at which the FWHM linewidth falls to half that of the PL spectrum.

\section{RESULTS AND DISCUSSION}

The absorption and PL spectra of F8BT:P3HT blends with different P3HT fraction are displayed in Figure 1. The pristine P3HT absorption spectrum possesses a main peak at 2.4 $\mathrm{eV}$ with two shoulders around 2.2 and $2.1 \mathrm{eV}$, respectively, which are characteristic of co-facial lamellar $\pi$-stacked P3HT aggregates. ${ }^{16}$ The blend absorption spectra is in turn composed of a broad absorption band $(2.7 \mathrm{eV})$ characteristic of F8BT and an additional shoulder near $2.3 \mathrm{eV}$.

Since the low energy band is not accounted by pristine polymer contribution, strong degree of $\pi-\pi$ interaction between host and guest polymers in blend is inferred. Wu et al. 

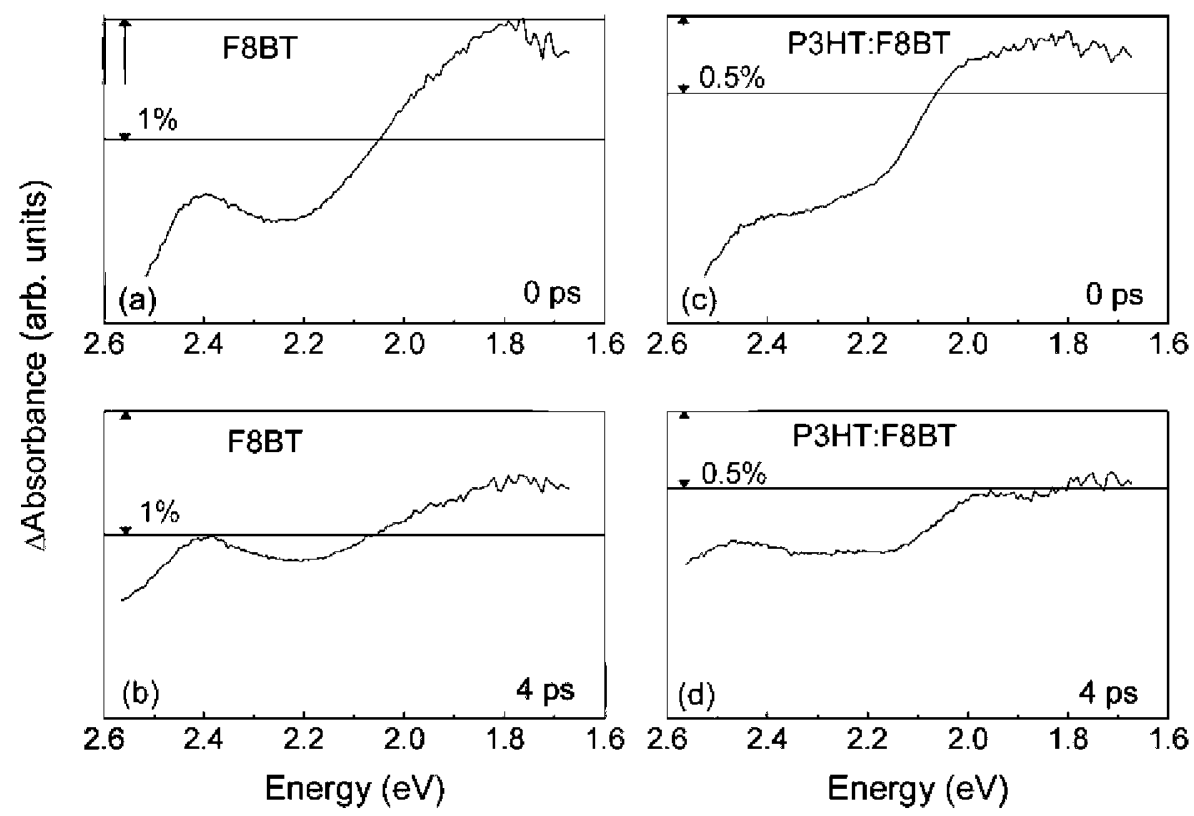

FIGURE 2 (a and b) $\Delta A$ spectra of F8BT at 0 and 4 ps delay, respectively. (c and d) $\Delta A$ spectra of F8BT:P3HT blends at 0 and 4 ps, respectively.

proposed recently that the strong affinity between the electron-donor thiophene and electron-acceptor benzothiadiazole groups present, respectively, in P3HT and F8BT could result in the formation of a F8BT:P3HT complex enabling wavefunction mixing of the ground and excited states. ${ }^{17}$ At high P3HT weight percentage (80\%), the spectrum evolves with an obvious long energy shoulder at $2.1 \mathrm{eV}$, indicating the appearance of P3HT aggregates in blend films. At lower P3HT dopings, Gaussian multipeak decomposition of the absorption spectra provides the separate spectral contributions of F8BT and F8BT:P3HT complexes in blends [Fig. 1(b)].

Parallel to the strengthening of the absorption shoulder with P3HT doping, the PL spectra of blends [Fig. 1(c)] evolve from F8BT green-yellow emission to a characteristic PL spectrum with maximum at 2.0 and shoulders at 1.8 and $1.7 \mathrm{eV}$. The enhanced tail of the PL at high P3HT doping could be related to inter-chain emissive species. The gradual quenching of F8BT fluorescence with P3HT doping and the concomitant augment of emission in the red part of the spectrum imply a FRET process between host and guest. Optimum Förster resonant conditions are supported by the large spectral overlap between F8BT emission and P3HT absorption. In films with P3HT fraction exceeding 15\%, the residual F8BT emission is no longer distinguished.

The FRET dynamics in blends were investigated in more details by aims of femtosecond transient absorption spectroscopy. The transient absorption spectra at 0 and 4 ps delays in pristine F8BT and a 5\% weight mixture of P3HT in F8BT are shown in Fig. 2. The 0 ps differential absorption $(\triangle A)$ spectrum of F8BT (Fig. 2(a)) is characterized from high to low energies by a negative photobleach (PB) tail extending down to $2.4 \mathrm{eV}$, a stimulated emission (SE) band centered at $2.2 \mathrm{eV}$ and positive $\Delta A$ between 2.0 and $1.7 \mathrm{eV}$ associated to photoinduced absorption (PA). Lack of spectral evolution is inferred from the 4 ps $\triangle A$ spectrum [Fig. 2(b)] except for a pronounced spectral dip at $2.4 \mathrm{eV}$, likely caused by a change in the relative concentration of singlet excitons (which dominates most of the pump-probe spectrum) respect to polaron pairs. This effect is emphasized upon increasing the pump fluence and consequently the polaronpair quantum yield. ${ }^{18}$ The 0 ps $\Delta A$ spectrum of the F8BT:P3HT blend, (Fig. 2(c)), preserves the spectral features characteristic of F8BT except for the absence of spectral dip at $2.4 \mathrm{eV}$, and a red-shift of the isosbestic point by about 20 meV. Note that the $3.2 \mathrm{eV}$ pump pulse implies that photoexcitation is placed mainly (not exclusively) in F8BT chromophores. Thus, the subtle spectral differences observed in blends could be associated to minor contributions from direct P3HT photoexcitation. At 4 ps delay [Fig. 2(d)], the positive PA band ascribed to F8BT is overcome by negative $\Delta A$ due to guest SE, (in the same spectral position as PL in blends). The emission build-up confirms that the FRET rate saturates in less than 4 ps. Additional insights into the efficiency of this process are displayed in Figure 3.

The $\Delta A$ dynamics at 2.3 and $2.1 \mathrm{eV}$ reflect slow-down and build-up kinetics respectively upon doping, concomitant with the appearance of the P3HT PB band [Fig. 3(a,b)]. Likewise, the $\Delta A$ dynamics at $1.8 \mathrm{eV}$ of 1 and $5 \%$ blends [Fig. 3(c)] show a change of sign as F8BT PA is gradually overcome by P3HT SE due to energy transfer. In $40 \%$ blends, the transfer rate approaches the pulse duration and gradual population effects are no longer visible. Interestingly, the SE contribution at $1.8 \mathrm{eV}$ is overwhelmed by a characteristic long-lived PA, absent in blends with low P3HT content. Global fit analysis involving three excited states associated to unrelaxed 

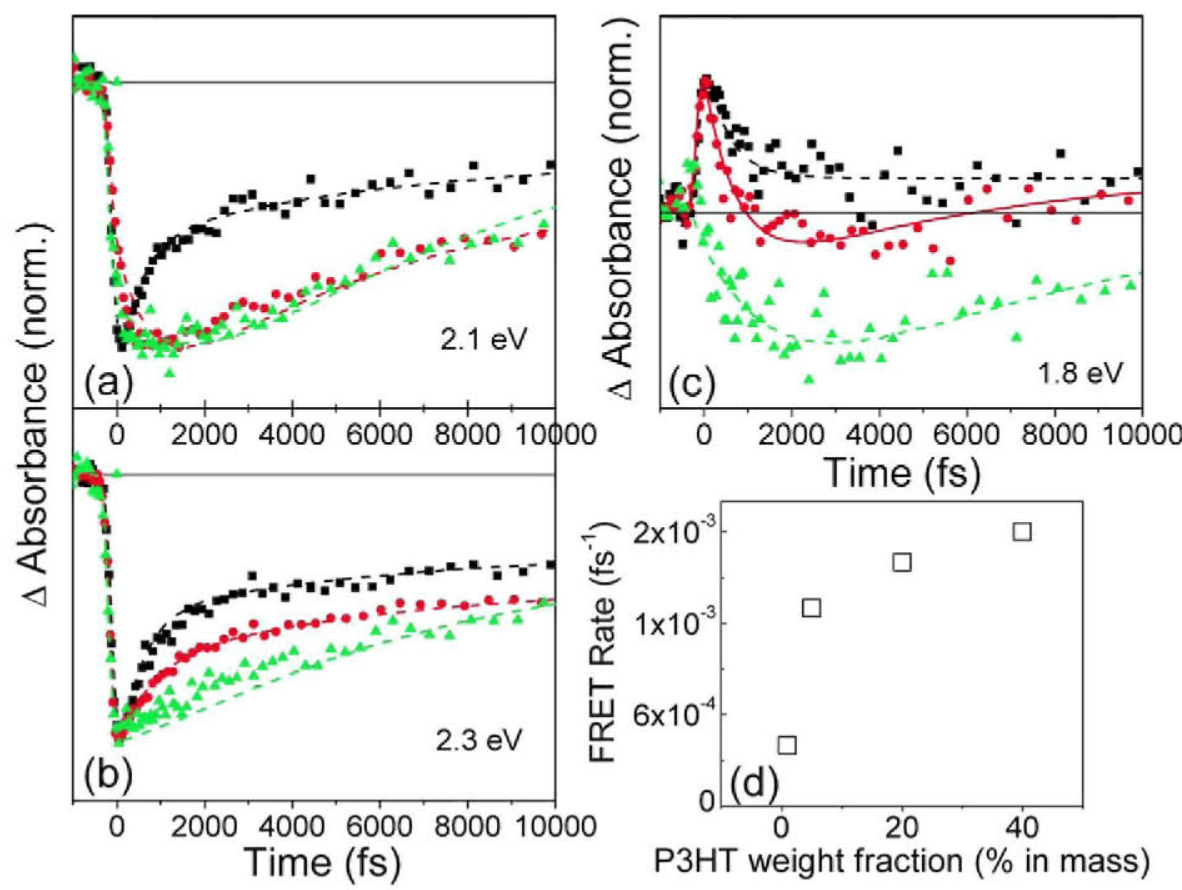

FIGURE 3 Pump-probe dynamics at $2.1 \mathrm{eV}$ (a), $2.3 \mathrm{eV}$ (b), and $1.8 \mathrm{eV}$ (c) of $1 \%$ (filled triangles), $5 \%$ (filled circles), and $40 \%$ blends (filled squares). Dashed lines stand for fits to kinetics. (d) FRET rates obtained from fits as function of P3HT concentration. [Color figure can be viewed in the online issue, which is available at wileyonlinelibrary.com.]

singlet excitons in F8BT $\left(S_{1}^{\mathrm{FBBT}}\right)$, P3HT $\left(S_{1}^{\mathrm{PHHT}}\right)$, and a relaxed excited state associated to $\mathrm{P} 3 \mathrm{HT}\left(X^{\mathrm{P} 3 \mathrm{HT}}\right)$ coupled by a sequential chain reaction $\left(S_{1}^{\mathrm{FBBT}} \rightarrow S_{1}^{\mathrm{P} 3 \mathrm{HT}} \rightarrow X^{\mathrm{P} 3 \mathrm{HT}}\right)$, was applied to fit the data.

The kinetic reaction (Scheme 1) involves direct photoexcitation of F8BT and sequential population of $S_{1}^{\text {P3HT }}$ and $X^{\mathrm{P} 3 \mathrm{HT}}$ exciton levels with the following rate equations:

$$
\begin{gathered}
\frac{d S_{0}^{F 8 B T}}{d t}=-g+k_{E T} S_{1}^{F 8 B T} \\
\frac{d S_{1}^{F 8 B T}}{d t}=g-k_{E T} S_{1}^{F 8 B T} \\
\frac{d S_{1}^{P 3 H T}}{d t}=k_{E T} S_{1}^{F 8 B T}-k_{1} S_{1}^{P 3 H T} \\
\frac{d X^{P 3 H T}}{d t}=k_{1} S_{1}^{P 3 H T}-k_{X} X^{P 3 H T}
\end{gathered}
$$

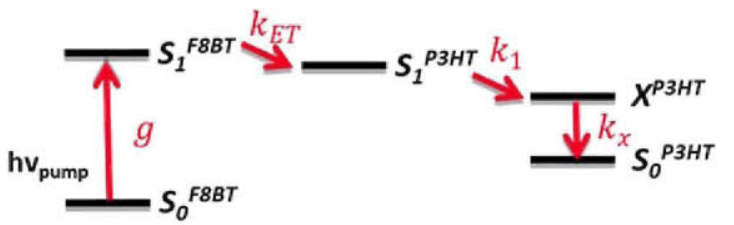

SCHEME 1 Energy levels and transitions diagram for F8BT:P3HT blends. [Color figure can be viewed in the online issue, which is available at wileyonlinelibrary.com.]

$$
\frac{d S_{0}^{P 3 H T}}{d t}=-k_{E T} S_{1}^{F 8 B T}+k_{X} X^{P 3 H T}
$$

where $S_{0}^{\mathrm{F} 8 \mathrm{BT}}, S_{0}^{\mathrm{P} 3 \mathrm{HT}}, g, k_{E T}, k_{1}$, and $k_{x}$ denote F8BT and P3HT ground states and the rates of pump-induced exciton generation, host:guest energy transfer, $X^{\mathrm{P} 3 \mathrm{HT}}$ generation rate and $X^{\mathrm{P} 3 \mathrm{HT}}$ decay to ground state respectively. Table 1 displays the parameters obtained from the fits.

The FRET rate values estimated from the build-up of P3HT exciton population are illustrated in Figure 3(d). A low content of P3HT in blend such is 5\% suffices to achieve large host:guest interaction reflected in a sub-picosecond energy transfer rate. FRET rates reach a maximum value at about $40 \%$ blend concentrations with a typical FRET timescale of 550 fs. This value confirms the strong FRET coupling present in these mixtures compared to other polymer:polymer blends. ${ }^{1}$ The gradual rate increase and absence of FRET rate saturation up to $40 \%$ guest weight content suggests a large degree of miscibility of P3HT in F8BT. In conjugated polymer

TABLE 1 Decay Rates Extracted from Global Fit Analysis of Pump-Probe Data

\begin{tabular}{llcc}
\hline $\begin{array}{l}\text { P3HT Weight } \\
\text { Fraction }(\%)\end{array}$ & $k_{\mathrm{ET}}\left(\mathrm{ps}^{-1}\right)$ & $k_{1}\left(\mathrm{ps}^{-1}\right)$ & $k_{x}\left(\mathrm{ps}^{-1}\right)$ \\
1 & $4 \times 10^{-1}$ & $8.3 \times 10^{-2}$ & $2.4 \times 10^{-3}$ \\
5 & 1.3 & $2.2 \times 10^{-1}$ & $1.3 \times 10^{-3}$ \\
20 & 1.6 & $9.4 \times 10^{-1}$ & $2.8 \times 10^{-3}$ \\
40 & 1.8 & $2.3 \times 10^{-1}$ & $5.1 \times 10^{-4}$
\end{tabular}



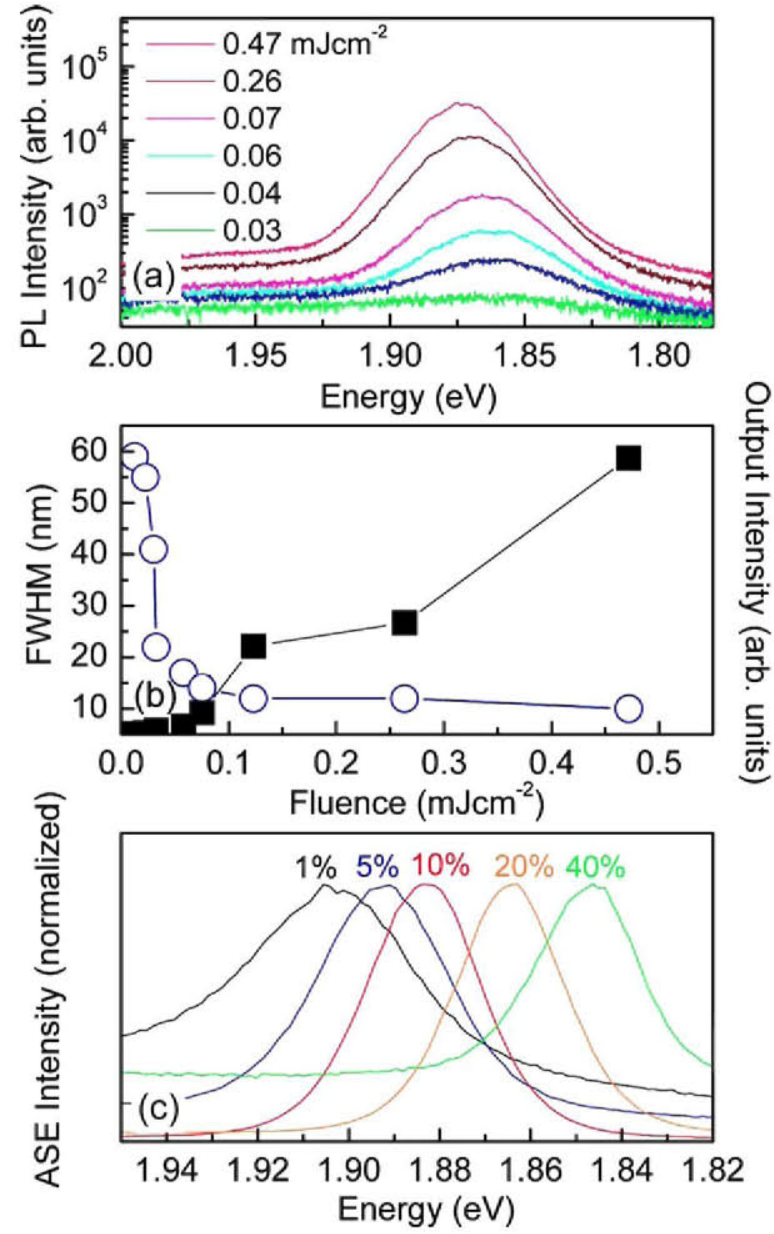

FIGURE 4 (a) ASE spectra and (b) corresponding values of FWHM (open circles, left ordinate) and integrated area (filled squares, right ordinate) as a function of input fluences, for a F8BT:P3HT (15:85) blend film pumped at $530 \mathrm{~nm}$. (c) Normalized ASE spectra at $450 \mathrm{~nm}$ photoexcitation of F8BT:P3HT blend films with 1, 5, 10, 20, and 40 wt \% P3HT compositions. [Color figure can be viewed in the online issue, which is available at wileyonlinelibrary.com.]

blends, FRET is often jeopardized by the appearance of polymer phase segregated domains at high guest content and consequently an enhancement of bulk emission. ${ }^{19}$ Next, the optical gain properties of F8BT:P3HT blends were studied by amplified spontaneous emission (ASE) measurements. Figure 4(a) shows the typical ASE spectra of a $15 \%$ blend upon $530 \mathrm{~nm}$ photoexcitation. At sufficiently high excitation densities, spectral narrowing is achieved with emission centered at around $1.9 \mathrm{eV}$ coinciding with the $0-1$ vibronic peak of the PL spectrum. Figure 4(b) shows the dependences of the FWHM ASE linewidth and output light intensity (integrated area) versus input pump fluence, respectively. ASE gives rise to a soaring growth of the output intensity at the highest gain position and a dramatic collapse in the linewidth from 60 to less than $10 \mathrm{~nm}$. Figure 4(c) displays the normalized ASE spectra of blends excited at $450 \mathrm{~nm}$. As the P3HT weight fraction increases, there is a slight red-shift of the
ASE peak from 1.9 to $1.85 \mathrm{eV}$ caused by the differences in dielectric constants of F8BT and P3HT in the red spectral region. ${ }^{14}$ Likewise, the strong fluorescence background seen in 1 and $5 \%$ blends is explained as due to residual F8BT emission, in agreement with PL measurements.

Hereafter, we evaluate and compare the impact of generation rate, (FRET-assisted versus direct excitation), on optical gain by performing ASE measurements on a set of blends pumping at 450 and $530 \mathrm{~nm}$ (predominant host or guest excitation, respectively). For comparative purposes, energy pulses employed for photoexcitation at 450 and $530 \mathrm{~nm}$ had same duration and were focused on approximately same areas.

ASE thresholds upon $530 \mathrm{~nm}$ initially drop with concentration, a behavior likely caused by the progressive absorption increase at $530 \mathrm{~nm}$ with P3HT doping [Fig. 5(a)]. Saturation and raise is followed at higher doping levels, which is tentatively assigned to counter-balancing concentration quenching effects, in line with the decrease in PL quantum efficiency at high guest doping reported in polymer blends elsewhere. ${ }^{17,20,21}$ The ASE thresholds upon $450 \mathrm{~nm}$ excitation follow a similar trend, the initial threshold decrease being associated in turn to FRETassisted exciton population. The measured thresholds at $450 \mathrm{~nm}$ are mostly below those at $530 \mathrm{~nm}$, observing the larger differences at low doping concentrations (1 and 5\%). For such low P3HT fractions, FRET contributes to reduce the ASE threshold by a factor of 3. The lowest ASE threshold values are obtained however at 20\% P3HT concentration $(0.05$ and 0.06 $\mathrm{mJcm}{ }^{-2}$ for 450 and $530 \mathrm{~nm}$ excitation, respectively). At those medium-high concentrations, the differences in ASE thresholds upon host/guest excitation shrink owing to the enhanced $530 \mathrm{~nm}$ photon absorption of blends. Thus, FRET appears to be particularly effective to reduce thresholds in blends with very low guest content.

In order to shed light into the FRET to ASE contribution, we compared the thresholds measured upon $450 \mathrm{~nm}$ excitation with those that would yield exclusively from direct guest photoexcitation at same wavelength, $\left(E_{\text {th }}^{\prime} 450\right)$. These latter values were estimated by taking into account the ASE threshold at $530 \mathrm{~nm}\left(\mathrm{E}_{\text {th }} 530\right)$ and the different absorbance values at $450\left(A_{450}\right)$ and $530 \mathrm{~nm}\left(A_{530}\right)$ as:

$$
E_{t h}^{\prime 450}=1.18 E_{t h}^{530} \frac{\left(1-10^{-A_{530}}\right)}{\left(1-10^{-A_{450}}\right)}
$$

where 1.18 is the $450-530 \mathrm{~nm}$ photon energy ratio, and $A_{450}\left(A_{530}\right)$ is determined from spectral decomposition of the blends absorption spectra into their guest and host components [Fig. 1(b) for details]. The $E_{\mathrm{th}}^{\prime} 450$ values exceed in all cases $E_{\mathrm{th}} 530$ by 1 order of magnitude [Fig. 5(a)] confirming that the contribution to ASE from direct guest photoexcitation at $450 \mathrm{~nm}$ is negligible.

Next, we quantify and compare the ASE efficiencies, determined as the absorbed photon density at threshold, upon host and guest photoexcitation, respectively. Bearing this in 

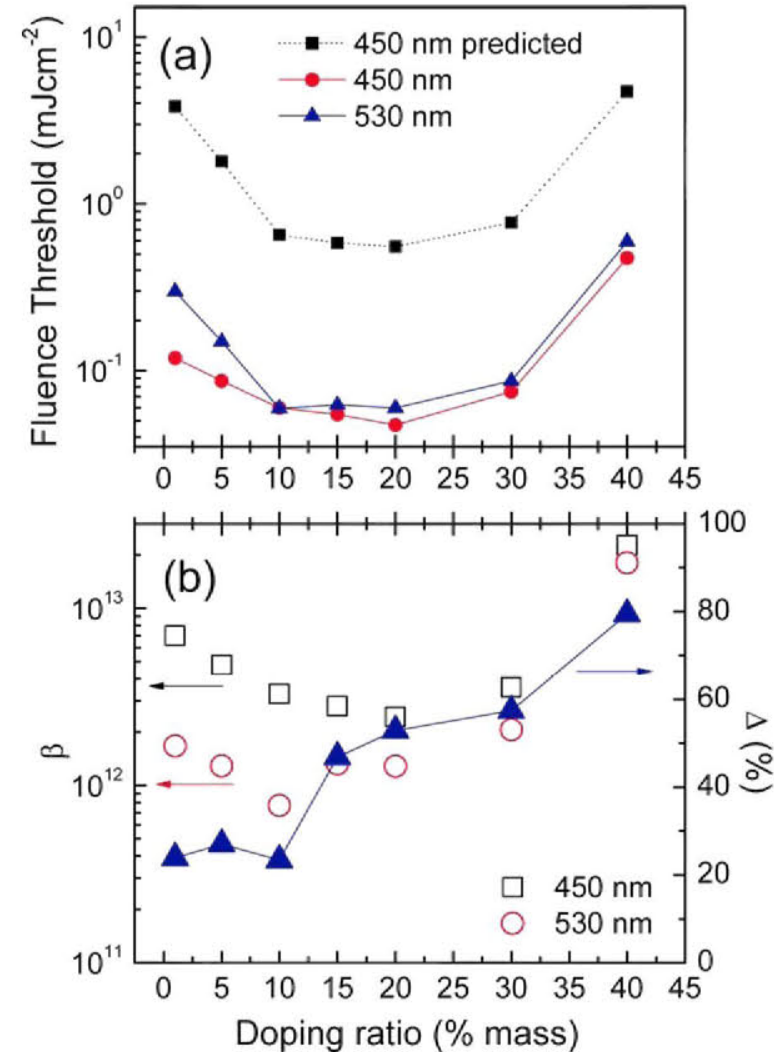

FIGURE 5 (a) ASE threshold values of P3HT/F8BT blend films as a function of P3HT concentration upon $450 \mathrm{~nm}$ (filled circles) and $530 \mathrm{~nm}$ (filled triangles) excitation. The predicted ASE thresholds from direct $\mathrm{P} 3 \mathrm{HT}$ photoexcitation at $450 \mathrm{~nm}$ for different P3HT doping are shown as filled squares. (b) The absorbed photon number at $450 \mathrm{~nm}$ (open squares) and $530 \mathrm{~nm}$ (open circles) and the FRET to ASE efficiency (filled triangles) as function of P3HT concentration. [Color figure can be viewed in the online issue, which is available at wileyonlinelibrary.com.]

mind, we define the total absorbed photon number at threshold conditions upon $\lambda$ excitation wavelength $(\beta)$ as:

$$
\beta \sim \frac{\lambda}{h c} S E_{t h} A
$$

where $S$ refers to the excitation area, $E_{\text {th }}$ is the measured ASE fluence threshold, and $A$ stands for the absorbance values of blends at $\lambda$ wavelength. The FRET-assisted ASE efficiency $(\Delta)$ is then defined from the ratio of the absorbed photon numbers at threshold upon $450\left(\beta_{450}\right)$ and $530 \mathrm{~nm}$ $\left(\beta_{530}\right)$ photoexcitation as:

$$
\Delta(\%)=100 * \frac{\beta_{530}}{\beta_{450}}
$$

The dependencies of $\beta_{450}, \beta_{530}$, and $\Delta$ with P3HT concentration are shown in Figure $5(\mathrm{~b})$. Interestingly, the $\beta_{450}$ values are above the $\beta_{530}$ for all concentrations, suggesting that a limited number of the total excitons harvested by F8BT will lead to guest optical gain. The differences diminish with concentration; in $40 \%$ blends for instance a $\Delta$ value of $80 \%$ is found, implying that $20 \%$ of the total absorbed photons by F8BT do not contribute to ASE. In the low doping regime, the progressive loss reduction upon increasing the P3HT weight fraction is concomitant with enhancement of FRET rate values and seems to suggest that losses stem mainly from inefficient energy transfer, as supported by the residual F8BT emission observed in blends with 1-15\% P3HT content. At high doping levels however, the lack of residual F8BT emission points towards a different exciton loss mechanism competing with FRET-assisted exciton population. It has been previously shown that exciton-exciton annihilation is particularly pronounced in F8BT owing to the large spectral overlap between the PL emission and excited state absorption, which yields to a large exciton-exciton interaction radius. ${ }^{22}$ Therefore, in the high concentration regime, exciton-exciton annihilation in F8BT could be the primary mechanism responsible for the different ASE efficiencies upon direct/indirect P3HT photoexcitation. At high P3HT content, competition between FRET and F8BT exciton-exciton annihilation leads to $20 \%$ of the total harvested F8BT excitons failing to contribute to ASE.

\section{CONCLUSIONS}

The ASE threshold values of conjugated polymer blends were assessed upon two different generation rate mechanisms: direct and FRET-assisted guest exciton generation. In the low guest concentration regime we observe large differences in the threshold values obtained upon these two photoexcitation schemes. Such differences are primarily caused by the large dilution and low absorption of guest polymer in blends, leading to differences of one order of magnitude in favor of FRET-assisted ASE. The ASE efficiencies obtained by accounting for the relative host (guest) absorption in blends indicate however supremacy of direct photoexcitation respect to FRET-assisted guest exciton generation, due to exciton losses competing with FRET. In blends with the highest FRET rates, losses amount to approximately $20 \%$ of the total harvested exciton population being likely attributed to exciton-exciton annihilation. Our results suggest that excitonic losses in strongly coupled host:guest systems upon large fluence photoexcitation conditions are not negligible and their hindrance would lead to further performance improvements of optically pumped lasers and optical amplifiers. These observations are particularly relevant in polyfluorenebased gain media, where excitonic losses upon high photoexcitation fluences are notorious.

\section{ACKNOWLEDGMENTS}

L. Wu and J. Cabanillas-Gonzalez are grateful to the China Scholarship Council for financial support through project number: 201206230083. J. Cabanillas-Gonzalez thanks the Spanish Ministry of Economy and Competitiveness for research funding through projects MAT2014-57652-C2-1-R (LAPSEN) and PCIN2015-169-C02-01 (MOFSENS). The authors thank the Madrid 
regional government under grant S2013/MIT-3007 (MAD2D project) for research funding.

\section{REFERENCES AND NOTES}

1 A. R. Buckley, M. D. Rahn, J. Hill, J. Cabanillas-Gonzalez, A. M. Fox, D. D. C. Bradley, Chem. Phys. Lett. 2001, 339, 331-336.

2 C. R. McNeill, N. C. Greenham, Adv. Mater. 2009, 21, 38403850.

3 S. Perissinotto, M. Garbugli, D. Fazzi, C. Bertarelli, M. Carvelli, A. R. S. Kandada, Z. N. Yue, K. S. Wong, G. Lanzani, Chem. Phys. Chem. 2011, 12, 3619-3623.

4 R. Gupta, M. Stevenson, A. J. Heeger, J. Appl. Phys. 2002, 92, 4874-4877.

5 M. Berggren, A. Dodabalapur, R. E. Slusher, Z. Bao, Nature 1997, 389, 466-469.

6 R. Gupta, M. Stevenson, A. Dogariu, M. D. McGehee, J. Y. Park, V. Srdanov, A. J. Heeger, H. Wang, Appl. Phys. Lett. 1998, 73, 3492-3494.

7 A. Camposeo, E. Mele, L. Persano, D. Pisignano, R Cingolani, Opt. Lett. 2006, 31, 1429-1431.

8 E. Engel, K. Leo, M. Hoffmann, Chem. Phys. 2006, 325, 170-177.

9 I. B. Greene, R. R. Millard, Phys. Rev. Lett. 1985, 55, 13311334.

10 T. Q. Nguyen, I. B. Martini, J. Liu, B. J. Schwartz, J. Phys. Chem. B 2000, 104, 237-255.
11 J. Cabanillas-Gonzalez, C. Sciascia, G. Lanzani, S. Toffanin, R. Capelli, M. C. Ramon, M. Muccini, J. Gierschner, T. Y. Hwu, K. T. Wong, J. Phys. Chem. B 2008, 112, 11605-11609.

12 D. Amarasinghe, A. Ruseckas, A. E. Vasdekis, G. A. Turnbull, I. D. W. Samuel, Adv. Mater. 2009, 21, 107-110.

13 Z. Yu, X. Guo, Q. Zhang, L. Chi, T. Chen, R. Xia, L. Wu, L. Lüer, J. Cabanillas-Gonzalez, J. Phys. Chem. C2016, 120, 11350-11358.

14 R. Xia, P. N. Stavrinou, D. D. C. Bradley, Y. Kim, J. Appl. Phys. 2012, 111, 123107, 1-8.

15 Y. Kim, D. D. C. Bradley, Curr. Appl. Phys. 2005, 5, 222-226.

16 J. Clark, J. F. Chang, F. C. Spano, R. H. Friend, C. Silva, Appl. Phys. Lett. 2009, 94, 163306.

17 L. Wu, S. Casado, B. Romero, J. M. Otón, J. Morgado, C. Müller, R. Xia, J. Cabanillas-Gonzalez, Macromolecules 2015, 48, 87658772.

18 C. Silva, A. S. Dhoot, D. M. Russell, M. A. Stevens, A. C. Arias, J. D. Mackenzie, N. C. Greenham, R. H. Friend, S. Setayesh, K. Mullen, Phys. Rev. B 2001, 64, 125211, 1-7.

19 R. Gupta, M. Stevenson, A. J. Heeger, J. Appl. Phys. 2002, 92, 4874-4877.

20 J. Cabanillas-Gonzalez, A. M. Fox, J. Hill, D. D. C. Bradley, Chem. Mater. 2004, 16, 4705-4710.

21 A. Camposeo, E. Mele, L. Persano, D. Pisignano, R. Cingolani, Phys. Rev. B 2006, 73, 165201.

22 M. A. Stevens, C. Silva, M. D. Russell, R. H. Friend, Phys. Rev. B 2001, 63, 165213,1-18. 